\title{
Prevención del suicidio: concepción de estudiantes universitarios
}

\author{
Suicide prevention: university \\ students' conception
}

\author{
Prevenção do suicídio: concepção \\ de estudantes universitários
}

M. Astrês-Fernandes ${ }^{a *}$, J. Soares e Silva $^{\mathrm{b}}$, C. da Conceição
Machado-Sousac ${ }^{c}$ A.G. Silva-Sád ${ }^{\mathrm{d}}$, E.A. Soares-Monteiro,
A.L. Castelo Branco- de Oliveira ${ }^{\mathrm{f}}$, A.R. de Sousa- Ibiapina ${ }^{\mathrm{g}}$

ORCID:

a 0ooo-0001-9781-0752 b 0000-0001-6544-9632 c 0000-0002-6766-0500

d $0000-0002-9520-8941$ e $0000-0001-7685-6145$ f $0000-0002-2634-0594$

g $0000-0003-1373-3564$

Departamento de Enfermería, Universidad Federal de Piauí-UFPI, Teresina, Piauí, Brasil

Recibido: 23 marzo 2019/Aceptado: 18 agosto 2019

\begin{abstract}
RESUMEN
Introducción: El ingreso en las Universidades representa un cambio en el estilo de vida social, económico, personal y mental en los discentes (estudiantes). A veces el proceso de habituación representa un factor estresante que puede culminar en sintomas de ansiedad y depresión, y representa un factor de riesgo para la salud mental.

Objetivo: Analizar la concepción de los discentes del Curso de Bachillerato en Enfermería, sobre la experiencia de vivenciar discusiones sobre la temática de suicidio.

Métodos: Estudio descriptivo-exploratorio de abordaje cualitativo realizado en una Universidad Federal del Nordeste de Brasil, cuyo manejo de la información se realizó con un software de procesamiento de datos. En la investigación participaron quince estudiantes
\end{abstract}


del curso Bachillerato en Enfermería que integraban el Proyecto de Intervención Ser, Saber, Escuchar, Vivir, en el cual se desarrollan acciones para los universitarios sobre prevención al suicidio y valorización de la vida.

Resultados: Los resultados fueron analizados a partir de la nube de palabras y la representación gráfica del análisis de similitud, se observó que la concepción de los discentes se resumió a las palabras Proyecto y Salud. La palabra proyecto se relacionó con la motivación y las experiencias de los discentes, y la palabra salud con el suicidio como problema de salud pública.

Conclusión: La discusión sobre el tema se muestra positiva en la Academia por favorecer un espacio de escucha y libertad de diálogo que proporciona alivio mental, contribuye a minimizar síntomas de ansiedad y depresión entre los discentes, y puede contribuir a reducir los índices de suicidio en la institución.

Palabras clave: Suicidio/prevención y control; estudiantes de enfermería; universidades; Brasil.

\section{ABSTRACT}

Introduction: Entering the university entails changes in the students' lifestyles regarding the social, economic, personal, and metal areas. Sometimes, the related habituation process can produce stress, which is externalized as anxiety and depression, and which can become a risk to the students' mental health.

Objective: To analyze the conception which students from a course of nursing have on experiencing discussions around suicide.

Methods: This is a descriptive-exploratory qualitative study carried out in a Federal University of Northeastern Brazil. Fifteen students from a course of nursing, who integrated a project to develop actions on suicide prevention and life valorization, participated in this study. The resulting information was produced using a data processing software.

Results: The conceptions were analyzed through a word-cloud and graphical representation of similarities methodology. The resulting combined conception was summarized by the words Project and Health, representing the students' motivation and experiences, and suicide as a health problem, respectively.

Conclusion: The corresponding free-dialog discussion on the issue provided mental relief among the students by minimizing their symptoms of anxiety and depression. This approach can contribute to reduce the suicide rates in the institution.

Keywords: Suicide/prevention and control; students, nursing; universities; Brazil.

\section{RESUMO}

Introdução: O ingresso nas universidades representa uma mudança no modo de vida social, econômico, pessoal e mental dos estudantes. Às vezes, o processo de habituação representa um estressor que pode culminar em sintomas de ansiedade e depressão e, desse modo, representar um fator de risco para saúde mental.

Objetivo: Analisar a percepção dos discentes sobre a experiência de vivenciar discussões sobre temática de suicídio.

Métodos: Trata-se de um estudo descritivo-exploratório, com abordagem qualitativa, realizado em uma Universidade Federal do Nordeste do Brasil. Participaram da pesquisa 15 estudantes do curso Bacharelado em Enfermagem que integravam o Projeto de Intervenção Ser, 
Saber, Ouvir, Viver, no qual eram desenvolvidas ações de prevenção ao suicídio e valorização da vida direcionado aos estudantes universitários da instituição.

Resultados: Os resultados foram analisados a partir a nuvemn da palavra e da representação gráfica de análise de similaridade, observou-se que a concepção dos alunos foi resumida às palavras Projeto e Saúde. A palavra projeto estava relacionada à motivação e as experiências dos alunos e a palavra saúde com suicídio como um problema de saúde público.

Conclusão: Portanto, o projeto em questão além de favorecer um ambiente de liberdade de diálogo no âmbito acadêmico, proporciona também alívio mental que, com o passar das reuniões, pode minimizar os sintomas depressivos e ansiosos que os discentes podem apresentar, em consequência, reduzindo os índices de suicídio na instituição.

Palavras chave: Suicídio/prevenção \& controle; estudantes de enfermagem; universidades; Brasil.

\section{INTRODUCCIÓN}

El ingreso a las Universidades representa una alteración relevante del estilo de vida para la mayoría de las personas, ya que el nuevo medio social evidencia otras exigencias de convivencia y responsabilidades ${ }^{1}$. De esta forma, las emociones experimentadas en este ambiente están íntimamente relacionadas con la adaptación y éxito académico, así como con la salud y el bienestar de los alumnos ${ }^{2}$.

El proceso de habituación, a veces puede ser aprehendido como un factor estresante e impactar directamente en la salud de los estudiantes, lo que puede desencadenar síntomas ansiosos y depresivos en los discentes, y así, representar un factor predisponente al suicidio ${ }^{3-5}$. Además, el inmediatismo, individualismo y la prisa, muy presentes en el contexto universitario, se muestran también como factores causales de la enfermedad psíquica ${ }^{3.4}$.

Por otra parte, las relaciones establecidas por los académicos pueden constituirse en un factor de riesgo o protección a la salud mental. De esta forma, tener apoyo social, poseer habilidades sociales e involucrarse en actividades recreativas son identificadas como variables de protección para la salud mental de los estudiantes. Las características de la vida académica y relacional son las que con mayor frecuencia se asocian a la presencia de sufrimiento psíquico entre los universitarios, lo que revela su importancia ${ }^{5-7}$.

En Brasil, las tasas de suicidio correspondieron en la última década, a un total de 5.7 casos por cada 100 mil habitantes y representaron la cuarta causa de muerte, entre las principales, en el grupo de edad de 15 a 29 años. De ahí la importancia de la planificación de intervenciones que contribuyan con el bienestar de los alumnos y vivencias más positivas en el ambiente de enseñanza, donde al mismo tiempo que se preparan, organizan, planifican y discuten eventos de artes y valorización a la vida, se ofrecen diversas perspectivas relativas al cuidado en salud, lo que también posibilita la creación de un ambiente en el cual las posiciones heterogéneas y consensos pueden ser debatidas, con el propósito de enriquecer la mirada y la práctica de las personas implicadas ${ }^{8,9,10}$.

En este sentido, en las Universidades se ha abordado la temática de suicidio y valorización de la vida. Por eso, el Proyecto Ser, Saber, Escuchar, Vivir vino como una propuesta de intervención para ampliar los conocimientos de la comunidad universitaria acerca del tema y sus facetas. Sin embargo, es importante señalar que es necesario conocer al estudiante que integra el proyecto para saber si este contribuye a su experiencia personal y profesional. 
Con el fin de apoyar el estudio, se utilizó la Teoría de los Gráficos para análisis de similitud y nube de palabras formadas por medio de un corpus textual de las palabras de los discentes integrantes del proyecto. Esta teoría se constituye de un modelo matemático que identifica las conexiones entre palabras y resultados, y así, auxilia en la identificación de la estructura del corpus textual insertado en el software para interpretación de los datos ${ }^{11}$. Por lo tanto, el objetivo del estudio consiste en describir la percepción de discentes integrantes del Proyecto Ser, Saber, Escuchar, Vivir, sobre la experiencia de vivenciar discusiones sobre la temática de suicidio.

\section{MÉTODOS}

Se trata de un estudio descriptivo-exploratorio, con abordaje cualitativo, realizado en una Universidad Federal del Nordeste de Brasil. La investigación tuvo la siguiente pregunta orientadora: ¿Cuál es la percepción de los discentes integrantes del Proyecto Ser, Saber, Escuchar, Vivir; sobre la experiencia de vivenciar discusiones sobre la temática de suicidio?

En la investigación participaron quince estudiantes del curso Bachillerato en Enfermería del tercero al octavo semestre que integraron el Proyecto de Intervención Ser, Saber, Escuchar, Vivir, en el cual se desarrollan acciones de prevención al suicidio y valorización de la vida, dirigidas a los estudiantes universitarios de la Institución. El proyecto se inició en 2016 motivado por algunos casos de suicidio en la Universidad, a partir de ese momento, los estudiantes y los docentes se sensibilizaron y decidieron comenzar este proyecto de valoración de la vida.

Las actividades desarrolladas por el proyecto englobaban ruedas de conversación, talleres terapéuticos, charlas, terapias comunitarias integrativas, entre otras, con una frecuencia mensual. Cabe señalar que para este estudio, se excluyeron los alumnos que no presentaron frecuencia continua en las reuniones del proyecto y los que no respondieron al contacto realizado por la coordinadora del proyecto para fines de recolección de datos.

La recolección ocurrió en el período de agosto a septiembre de 2018, se utilizó un guion de entrevista semiestructurada, con preguntas abiertas sobre la experiencia del alumnado en el proyecto, así como datos sociodemográficos. El instrumento de recolección de datos fue construido por la propia investigadora responsable que también es coordinadora del proyecto, el cual no pasó por las etapas de validación. Las preguntas guía del enfoque a los entrevistados fueron: ¿Inicialmente, qué motivó su participación en este Proyecto de Valorización de la Vida?; ¿Qué agregó a su vida académica y profesional el Proyecto Ser, Saber, Escuchar, Vivir?; De las actividades que fueron desarrolladas, ¿cuál fue la que más le marcó a usted y el por qué?; ¿Qué propone como posibles intervenciones futuras, que puedan ser implementadas en la Institución (Departamento / Universidad), relacionadas a la prevención del suicidio y la valorización de la vida?; ¿Cómo se autoevalúa antes y después de la realización del proyecto relacionado al tema valorización de la vida y prevención al suicidio?

Después de la recolección, todas las entrevistas fueron transcritas y analizadas a través de un software para análisis de datos cualitativos, IRaMuTeQ (Interfaz de R pour les Analyses Multidimensionnelles de Textes et de Questionnaires). Este software permite diferentes formas de análisis estadísticos sobre el corpus textual, en cuanto al análisis de datos del presente estudio se utilizó el análisis de similitud y nube de palabras integradas a la herramienta ${ }^{12}$.

El análisis de similitud permite identificar las ocurrencias entre las palabras y los resultados al demostrar la conexión entre las palabras. A su vez, la nube de palabras agrupa las palabras y las organiza en función de la frecuencia presentada, y de esa forma atraviesa una idea de organización y de lectura más simplificada sobre los principales puntos del discurso ${ }^{12}$. 
El proyecto y la investigación en cuestión corresponden a un plan de trabajo de un macroproyecto sobre la prevalencia de síntomas ansiosos y depresivos en universitarios de una institución pública, aprobada por el Comité de Ética en Investigación de la Universidad Federal de Piauí, bajo el parecer de número 1.665. 294, el 5 de agosto de 2016. En todas las etapas del estudio se respetaron los principios éticos contenidos en la Resolución 466/2012 del Consejo Nacional de Salud, que reporta sobre los aspectos éticos y legales de investigación que involucra seres humanos. Todos los participantes firmaron los términos del Consentimiento Libre e informado.

\section{RESULTADOS}

El análisis de similitud o de semejanzas se constituye de un método de evaluación sostenido por medio de la Teoría de los grafos, cuya, técnica permite identificar las coyunturas. El resultado indica la conexión entre las palabras, y auxilia de esa forma, la identificación de la estructura de representación del corpus textual. (Figura 1)

A partir de la representación gráfica de la Figura 1, el software muestra como descripción las palabras más frecuentes que fueron: proyecto, vida, suicidio, acción, actividad y salud. Estas palabras corresponden a los puntos más fuertes observados en las conversaciones de los participantes, por medio de ellas, es posible establecer conexiones y transcribir lo que los participantes perciben en cuanto al proyecto.

En lo que se refiere al método de análisis de la nube de palabras, la agrupación y organización gráfica de estas ocurre en función de su repetición, lo que proporciona una rápida identificación de las palabras clave en la figura, como se puede ver en la Figura 2.

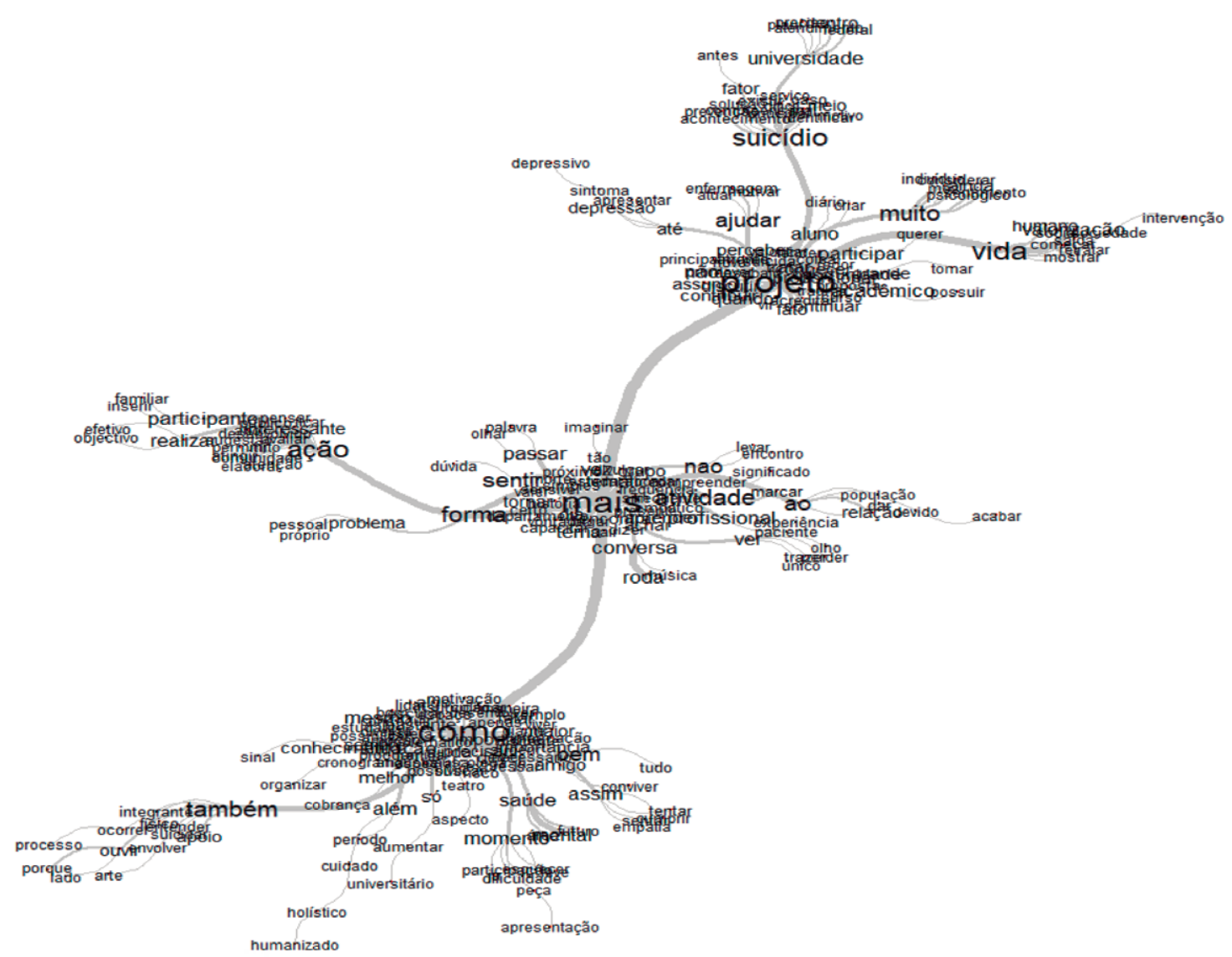

Figura 1. Análisis de similitud entre las palabras. Teresina, PI. Brasil, 2018. 


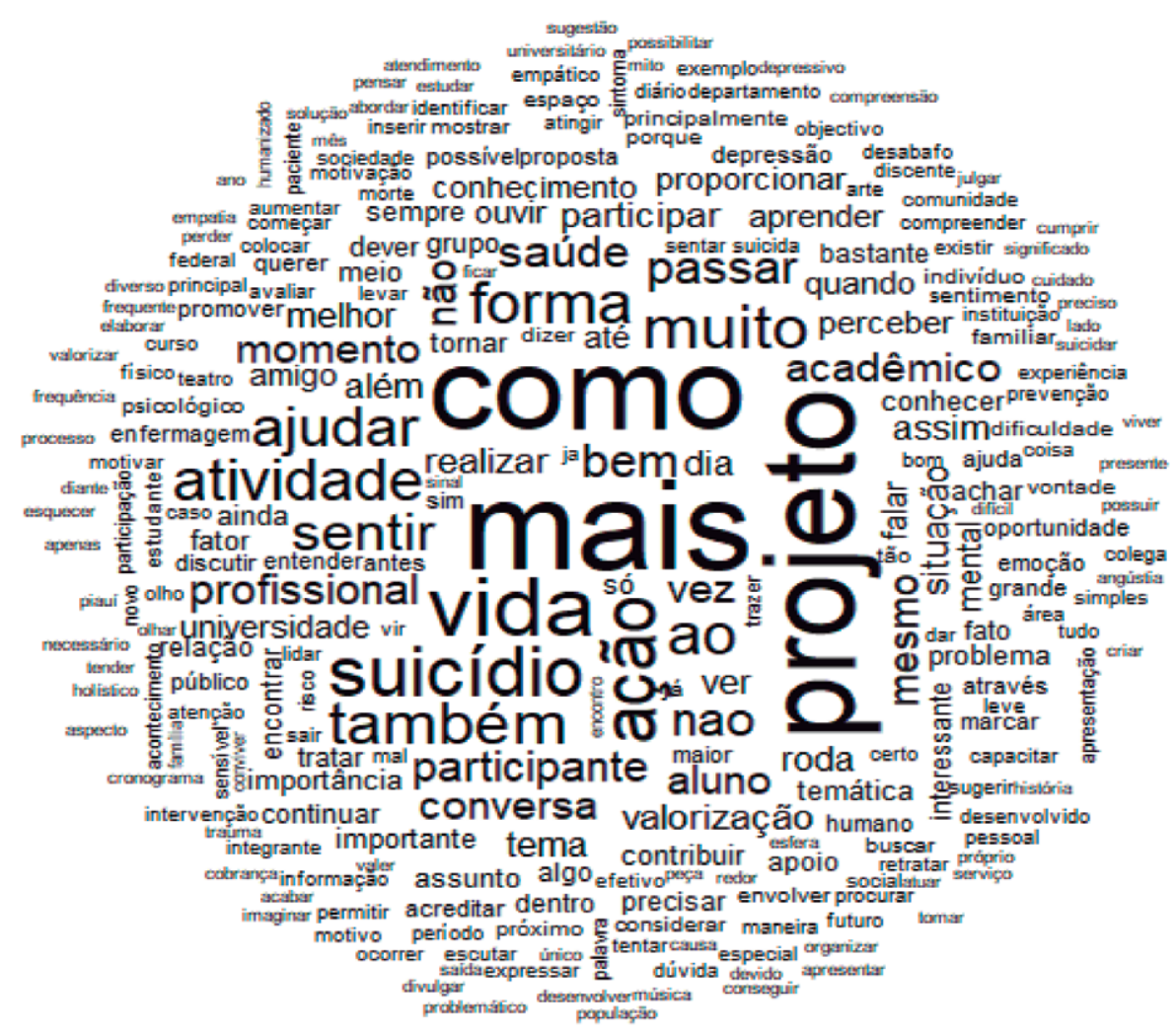

Figura 2. Nube de palabras. Teresina, PI. Brasil. 2018.

A partir de la representación con la nube de palabras se puede ver que el rango semántico de las palabras más frecuentes fue: cómo, proyecto, acción, vida, suicidio, ayuda, forma y actividad; mismas que pueden ser verificadas por la longitud mostrada en la representación gráfica. La idea de asociar palabras con las percepciones de los participantes sobre el proyecto a través de la observación de las más repetidas, se asemeja al análisis de similitud ya que la nube de palabras complementa la idea de asociación propuesta por la técnica de análisis de similitud, por lo tanto, hace la interpretación más clara y más concisa.

\section{DISCUSIÓN}

El Proyecto Ser, Saber, Escuchar, Vivir permitió a los estudiantes y maestros involucrarse en la creación de una nueva visión de la salud mental de las personas. Los integrantes de la comunidad se volvieron más sensibilizados, más atentos y más proactivos para desarrollar una cultura que favoreciera la comprensión de las necesidades psíquicas y emocionales de los estudiantes de la institución, aligerando el ambiente académico y corroborando la difusión de información sobre el tema a la comunidad universitaria. No obstante, para comprender mejor lo que pensaban los estudiantes participantes en el proyecto, se planteó este estudio exploratorio.

Al analizar la nube de palabras y la representación gráfica del análisis de similitud entre las palabras, se observaron de forma general las percepciones de los discentes frente a la vivencia en el proyecto de valorización de la vida. Cada palabra destacada indicó repeticiones en las palabras, y en consecuencia, significados semejantes. Sin embargo, algunas palabras llaman la atención ya que sus significados son más complejos, tales como: proyecto y salud. 
La palabra proyecto a menudo repetida, está relacionada con la motivación, metas y las experiencias de los estudiantes. La principal motivación de los participantes se refiere a la propuesta del proyecto, el cual proporcionó la creación de un espacio para discusión y realización de actividades relacionadas con temas tabúes, lo que trae un mayor conocimiento sobre las temáticas de suicidio y valorización de la vida, así como otros temas relacionados, como ansiedad, depresión y salud mental.

Con respecto a la palabra salud, los participantes apuntaron que el suicidio es un problema de salud pública y se enfatiza la importancia de la preparación de los profesionales de la salud sobre la temática en su proceso formativo. En efecto, el enfermero debe estar calificado y preparado para identificar las características que el individuo con potencial suicida presenta, como pensamientos y actitudes que evidencian desesperanza, desesperación y desamparo ${ }^{13}$.

En cambio, aunque la vivencia no sea la misma, se advierte que hay un déficit de conocimiento de los alumnos desde los primeros semestres hasta los últimos del curso de graduación. La literatura apunta, además, la falta de preparación y la poca exposición educativa en lo que se refiere al suicidio en cursos de graduación en el área de la salud y resalta la importancia de inversiones en actividades de intervención relacionadas al suicidio para profesionales de la salud desde la Academia ${ }^{14,15}$.

Para los discentes, el espacio teórico y físico donde el proyecto actuó, también sirvió como un lugar para desahogo de las angustias vivenciadas por ellos. El ambiente académico como espacio dinámico de relaciones, alegrías y conquistas es compartido, sin embargo; también hay en él secretos, angustias, sufrimiento y frustraciones, que son en la mayoría de las veces escondidas por jóvenes que perdieron o que pierden diariamente la esperanza y la expectativa de futuro. Bajo la influencia de esos pensamientos, algunos discentes ven en el suicidio la única posibilidad de liberación ${ }^{16}$.

Es válido recordar que en la transición hacia la Enseñanza Superior se verifica una mayor incidencia de problemas adaptativos al espacio académico, en consonancia con las dificultades de relación interpersonal y demandas personales que exigen del estudiante habilidades emocionales para superar el proceso sin que ocurran daños. Al estudiante se le exige el control de muchas demandas: familiar, personal, académica, social y profesional, que lo sobrecargan y le ocasionan sufrimiento psíquico ${ }^{17}$.

En este sentido, se cree en la urgencia de la necesidad de implementación de actividades de intervención en las instituciones de enseñanza superior, por medio de acciones eficaces que actúen como medida preventiva al suicidio en el medio académico. Se resalta que las actividades del Proyecto Ser, Saber, Escuchar, Vivir se realizan en forma de ruedas de conversación, talleres terapéuticos, terapias comunitarias, conferencias, teatro y musicales. Estas acciones fueron consideradas importantes por los participantes, pues promovieron momentos de conocimiento, reflexiones y bienestar. Esto se percibió cuando se visualizaron los términos: acción, vida y actividad en las representaciones gráficas de las Figuras 1 y 2.

Es necesario hacer hincapié en que las acciones de valorización de la vida necesitan involucrar a todos los que participan en el contexto, volviendo al fortalecimiento de la autoestima y autoimagen positivas. De esta forma, para desarrollar la comprensión es necesaria la expresión de su afecto, sea de cualquier naturaleza ${ }^{16}$.

En cuanto a los cambios, los participantes relataron que el proyecto promovió una visión empática y con menos juicios acerca del suicidio y la importancia de un cuidado holístico y humanizado para con los individuos con ideas suicidas. Aunque es sabido que los cambios ocurren a largo plazo y necesita tiempo de seguimiento para la observación, esa visión a corto plazo puede reflejar las bases para cambios futuros. 
Por consiguiente, se destaca que los enfermeros desempeñan un importante papel en la prevención y cuidado relacionados con el suicidio, y la formación de estos profesionales necesita revisión y perfeccionamiento para adecuar las líneas de cuidado a las vulnerabilidades personales. La formación de los profesionales de la enfermería necesita abarcar habilidades relacionadas al autoconocimiento, empatía, comprensión, comunicación, actitudes y conocimientos sobre los comportamientos suicidas, aunado a la posibilidad de prevención y esclarecimiento del papel de cada profesional en el cuidado a esos pacientes ${ }^{18,19}$.

\section{CONCLUSIÓN}

Las técnicas de análisis utilizadas brindaron una visión general gráfica de los conceptos centrales y sus conexiones con otros elementos, que permitieron a su vez el análisis de las concepciones de los discentes en cuanto a las vivencias durante el período de vigencia del Proyecto Ser, Saber, Escuchar, Vivir se resumieron a dos palabras: Proyecto y Salud.

La palabra proyecto se relacionó con la experiencia en cuanto a las actividades desarrolladas y al foco del proyecto, en reconocer el suicidio como problema de salud pública, y de esa forma, establecer acciones dirigidas a la valorización de la vida en el ambiente académico; lo que confirma que los objetivos del programa están encaminados adecuadamente. Es importante recordar que los participantes en esta investigación son estudiantes de enfermería y desde antes de su ingreso están orientados al cuidado de la salud y la vida, no obstante, este proyecto reforzó su convicción de cuidar la salud y la vida.

Cabe señalar que los estudiantes y los maestros se sintieron más conscientes de los problemas relacionados con este tema después de participar en este proyecto, ya que antes, muchos vieron con prejuicio el debate sobre este tema. Además, se convirtieron en multiplicadores del conocimiento sobre el suicidio y la apreciación de la vida.

Por lo tanto, promover la discusión del tema en el escenario universitario favorece un ambiente de intercambio de informaciones y libertad de diálogo; proporciona alivio mental y bienestar a los discentes por funcionar como un canal de escucha y apoyo, que contribuye a minimizar los síntomas depresivos y ansiosos y, en consecuencia, reducir los índices de suicidio en la institución.

Los trabajos de intervención de esta naturaleza son importantes en las Instituciones de Enseñanza Superior, teniendo en cuenta las altas tasas de suicidio entre el público joven. Los ejemplos de intervenciones que pueden implementarse en Instituciones de Educación Superior incluyen círculos de conversación, espacios de bienestar, talleres terapéuticos y servicios de apoyo psicológico.

Conforme a lo enunciado el proyecto ha logrado sensibilizar a la comunidad universitaria y se ha constituido en un espacio para el dialogo y expresión de inquietudes emocionales. Sin embargo, consideramos que la siguiente fase de este programa sería evaluar en forma más precisa cada una de las modalidades de intervención consideradas en el Proyecto para identificar aquellas que deban mejorarse o la inclusión de otras pertinentes.

\section{RESPONSABILIDADES ÉTICAS}

Protección de personas y animales. Los autores declaran que para esta investigación no se han realizado experimentos en seres humanos ni en animales.

Confidencialidad de los datos. En este artículo no aparecen datos confidenciales de los participantes. Derecho a la privacidad y consentimiento informado. Todos los participantes firmaron los términos del Consentimiento Libre e informado. 
Financiación. Ninguno.

Conflicto de Intereses. No hay conflicto de intereses entre los autores.

\section{REFERENCIAS}

1. Newman I, Ding L, Feng Y. Estimate of undergraduate university student alcohol use in China: a systematic review and meta-analysis. Arch Public Health 2017; 75(52):1-13. https://doi.org/10.1186/s1369o-017-0220-x

2. Ribeiro IJS, Pereira R, Freire IV, Oliveira BG, Casotti CA, Boery EN. Stress and Quality of Life Among University Students: A Systematic Literature Review. Health Prof. Educ 2018; 4(2):70-7. https://doi.org/10.1016/j.hpe.2017.03.002

3. Ariño DO,Bardagi MP. Relação entre Fatores Acadêmicos e a Saúde Mental de Estudantes Universitários. Psicol Pesq 2018; 12(3): 44-52. http://dx.doi.org/10.24879/2018001200300544

4. Fernandes MA, Vieira FER, Silva JS, Avelino FVSD, Santos JDM. Prevalence of anxious and depressive symptoms in college students of a public institution. Rev Bras Enferm. 2018; 71(suppl 5):2169-2175. http://dx.doi.org/10.1590/0034-7167-2017-0752

5. Ortega-Ceballos PA, Terrazaz-Meraz MA, Arizmendi-Jaime ER, Tapia-Domínguez M. Conocimientos, actitudes y factores asociados al consumo de tabaco en estudiantes universitarios de enfermeira. Enferm. univ. 2018; 15(2):159-71. http://dx.doi.org/10.22201/eneo.23958421e.2018.2.65173

6. Barbosa-Marendino R, Cardoso-Lisbôa HC, Silva-de Lima JP. Produtivismo acadêmico e percepções sobre qualidade de vida dos estudantes de pedagogia da UFF Niterói. Movimento-Revista de Educação 2018; 5(9):165-91. https://doi.org/10.22409/mov.voig.476

7. Graner KM, Ramos-Cerqueira ATA. Revisão integrativa: Sofrimento psíquico em estudantes universitários e fatores associados. Ciênc. saúde colet 2019; 24(4):1327-46.

http://dx.doi.org/10.1590/1413-81232018244.09692017

8. Secretaria De Vigilância em Saúde-Ministério da Saúde Brazil. Suicídio: Saber, Agir e Prevenir. Boletim Epidemiológico 2017; 48(30):1-15 https://bit.ly/2WoISGY

9. Imbrizi JM, Moreira MIB, Kinker FS. Da aula aberta à universidade aberta: reflexões sobre saúde mental, arte \& território. Gerais: Ver. Interinst. Psicol. 2019; 12(1):175-89. https://bit.ly/2PtrH5N

10. Leme VBR, Chagas APS, Carvalho AP, Padilha AP, Alves AJCP, Rocha CS. Habilidades Sociais e Prevenção do Suicídio: Relato de Experiência em Contextos Educativos. Estud. pesqui. psicol. 2019;19(1): 28497. https://doi.org/10.12957/epp.2019.43020

11. Donato SP, Ens RT, Favoreto EDA, Pullin EMMP. From similitude analysis to focal group: strategies for studies in the structural approach to social representations. Rev. Educ. Cult. Contemp 2017;14(37): 367-94. https://bit.ly/2Jxpxym

12. Souza MAR, Wall ML, Thuler ACMC, Lowen IMV, Peres AM. El empleo del software IRAMUTEQ en el análisis de datos en investigaciones cualitativas. Rev esc enferm USP. 2018; 52: eo3353. http://dx.doi.org/10.159o/S1980-220X2017015003353

13. Smith AR, Silva C, Covington DW, Joiner TE. An assessment of suicide-related knowledge and skills among health professionals. Health Psychol 2014; 33(2):110-9. https://doi.org/10.1037/aoo3106

14. Ferreira KG, Gonçalves MV. The students' perspective on suicide approach during Occupational Therapy training. Cad. Bras.Ter.Ocup. 2018; 26(4): 883-91. http://dx.doi.org/10.4322/2526-8910.ctoao1610

15. Reisdorfer N, Araújo GM, Hildebrandt LM, Gewehr TR, Nardino J, Leite MT. Suicídio na voz de profissionais de enfermagem e estratégias de intervenção diante do comportamento suicida. Revista de Enfermagem da UFSM 2015; 5(2):295-304. http://dx.doi.org/10.5902/2179769216790 
16. Martins FRS, Brito MA. Educação e Saúde Mental: Caminhos para a prevenção do suicídio. Diálogos e contrapontos: estudos interdisciplinares. 2017; 1(2): 18-30. https://bit.ly/36j4iK3

17. Nascimento IMC, Ruíz-Díaz Morales D. Comportamento suicida em estudantes de enfermagem do Campus Senador Helvídio Nunes de Barros; Universidade Federal do Piauí. Academo. 2016; 3(1):1-18. https://bit.ly/2Jzl6mt

18. Moraes S, Magrini DF, Zanetti ACG, Santos MAS, Vedana KGG. Atitudes relacionadas ao suicídio entre graduandos de enfermagem e fatores associados. Acta Paul Enferm 2016; 29(6): 643-9. http://dx.doi.org/10.1590/1982-019420160009o

19. Crespo-Knopfler S, González-Velázquez MS, Cuamatzi-Peña MT, Domínguez-Villanueva LD, GonzálezNila LI. Percepción de empleadores sobre el Licenciado en Enfermería de la Facultad de Estudios Superiores Zaragoza. Enferm univ. 2009; 6(1):7-11.https://doi.org/10.22201/eneo.23958421e.2009.1.341 